\title{
ON THE MAXIMAL ERGODIC THEOREM FOR ABEL-LIMITS ${ }^{1}$
}

\section{GIAN-CARLO ROTA}

The powerful and ingenious method of Chacón and Ornstein [1], later simplified by E. Hopf [6], has led to a proof of the ergodic theorem for general operators which is in many ways definitive. The purpose of this note is to show that if Abel convergence is used instead of Cesàro convergence, then a proof of the corresponding limit theorem is obtained which is of extreme simplicity. The argument below shows that Abel convergence, rather than Cesàro convergence, is the natural limiting process to be associated with the method of Chacón-Ornstein-Hopf.

Our simplification of Hopf's proof can be given in a few lines; we follow some of the steps in Hopf's basic Lemma 1. The proof given below can however be read without knowledge of Hopf's paper.

Let $T$ be a positive operator $(T f \geqq 0$ if $f \geqq 0)$ in $L_{1}(S, \Sigma, \mu)$ and $L_{\infty}(S, \Sigma, \mu)$, where $(S, \Sigma, \mu)$ is an arbitrary measure space. Assume that $T$ is a contraction $(\|T\| \leqq 1)$ in the first space, and that it is bounded in the second. The Abel-ergodic theorem states that for every $f$ in $L_{p}(S, \Sigma, \mu), 1<p<\infty$, the limit

$$
\lim _{\lambda \rightarrow 1}(1-\lambda) \sum_{n=0}^{\infty} T^{n} f(s) \lambda^{n}=\lim _{\lambda \rightarrow 1}(1-\lambda) R_{\lambda} f(s)
$$

exists for almost all $s$ in $S$. The associated Abel-maximal theorem states that the function

$$
f^{*}(s)=\sup _{0<\lambda<1} \sum_{n=0}^{\infty} T^{n} f(s) \lambda^{n}
$$

is of class $L_{p}$ whenever the function $f$ is of class $L_{p}, \infty>p>1$. The maximal theorem can be used to prove the Abel-ergodic theorem by using standard techniques (cf. Dunford-Schwartz [3, Chapter VIII], and Hille-Phillips [5, Chapter 18]). Equally standard techniques (cf., for example, Dunford-Schwartz, loc. cit., Theorem VIII. 6.8, which is easily adapted to Abel limits) show that the Abel-maximal theorem depends on the following weak-type $L_{1}$ estimate:

Basic Lemma. Let $T$ be a positive contraction operator in $L_{1}(S, \Sigma, \mu)$, and let $f$ be an integrable real function on the measure space $(S, \Sigma, \mu)$. Let $A=\left\{s \mid \sup _{0<\lambda<1} R_{\lambda} f(s)>0\right\}$. Then $\int_{A} f(s) \mu(d s) \geqq 0$.

Received by the editors July 30, 1962 .

${ }^{1}$ By contract with grant NSF-GP-149 and the Office of Naval Research. 
It is in proving this lemma (or variants thereof) that the difficulty of the ergodic theorem lies. The following is a short proof. We omit the argument $s$ in functions $(f(s)=f)$, unless it is indispensable. Following E. Hopf, we define the functions $h_{n}$ by the recursion

$$
h_{n+1}=T h_{n}^{+}-\overline{h_{n}}, \quad h_{0}=f,
$$

where $f^{+}=\max (f, 0)$ and $-f^{-}=\min (f, 0)$. It is obvious that $h_{n+1}^{-}$ $\leqq h_{n}^{-}$. Letting $h^{ \pm}(\lambda)=\sum_{n=0}^{\infty} h_{n}^{ \pm} \lambda^{n}$, we obtain the equation

$$
(I-\lambda T) h^{+}(\lambda)=f+(1-\lambda) h^{-}(\lambda),
$$

that is, shuffling terms,

$$
(1-\lambda)^{-1} f=(1-\lambda T)(1-\lambda)^{-1} h^{+}(\lambda)-h^{-}(\lambda) .
$$

Taking coefficients of the power series we see that $f=\sum_{0}^{n} h_{\mathbf{k}}^{+}$ $-T \sum_{0}^{n-1} h_{\mathbf{k}}^{+}-h_{n}^{-}$whence $\int f \geqq-\int h_{n}^{-}$(using the fact that $\int T g \leqq \int g$ for all positive $g$ ). But $\int_{A} f \geqq \int f$, since $f$ is negative on the complement of $A$, hence the proof will be complete if we can show that $h_{n}^{-}$decreases to zero, or, equivalently (since $h^{+} h^{-}=0$ ), that for every point $s$ in $A$, there is an $n$ for which $h_{n}^{+}(s)>0$. But this is a trivial consequence of the main hypothesis! Indeed, from $\left(^{*}\right)$ we get, dropping a positive term, $(I-\lambda T) h^{+}(\lambda) \geqq f$, whence, inverting (the inverse exists and preserves order for $0<\lambda<1$ since $T$ is a positive contraction) $h^{+}(\lambda) \geqq \sum_{n=0}^{\infty} T^{n} f \lambda^{n}$. The hypothesis now gives $\sup _{0<\lambda<1} h^{+}(\lambda)>0$ on $A$, so that for every $s$, some coefficients $h_{n}^{+}(s)$ must be positive, q.e.d.

It would be interesting to use the same method to give a proof of the Abel analog of Chacón's ergodic theorem [2].

\section{REFERENCES}

1. R. Chacón and D. Ornstein, $A$ general ergodic theorem, Illinois J. Math. 4 (1960), 153-160.

2. R.Chacon, An ergodic theorem for operators satisfying norm conditions, J. Math. Mech. 11 (1962), 165-172.

3. N. Dunford and J. Schwartz, Linear operators. I, Interscience, New York, 1958.

4. P. R. Halmos, Lectures on ergodic theory, The Mathematical Society of Japan, 1956 (reprinted by Chelsea, New York, 1960).

5. E. Hille and R. S. Phillips, Functional analysis and semigroups, Amer. Math. Soc. Colloq. Publ. Vol. 31, rev. ed., Amer. Math. Soc., Providence, R. I., 1957.

6. E. Hopf, On the ergodic theorem for positive linear operators, J. Reine Angew. Math. 205 (1960), 101-106.

7. K. Jacobs, Neuere Methoden und Ergebnisse der Ergodentheorie, Springer, Heidelberg, 1960.

MASSACHUSETTS INSTITUTE OF TEChNOLOGY 